\title{
Generalizations of some contractions in b-metric-like spaces and applications to boundary value problems
}

Kastriot Zoto ${ }^{1}\left(\mathbb{D}\right.$, Hassen Aydi ${ }^{2,3,4}$ and Habes Alsamir ${ }^{5^{*}}$

"Correspondence:

habes@dau.edu.sa

${ }^{5}$ College of Business

Administration-Finance

Department, Dar Al Uloom

University Saudi Arabia, Riyadh,

Saudi Arabia

Full list of author information is

available at the end of the article

\section{Springer}

\begin{abstract}
This paper provides two wide classes of contractions, which are obtained by using notions of $\alpha_{s^{p}}$-admissibility and the rich set of C-class functions in the setting of a complete $b$-metric-like space under more general contractive conditions. An application is provided and many known results in the literature can be derived.
\end{abstract}

MSC: $47 \mathrm{H} 10 ; 54 \mathrm{H} 25$

Keywords: $\alpha_{s^{p}}$-admissible pair; $\alpha_{S^{p}}-(C, N, \Theta)$ contraction; $\alpha_{S^{p}}-(I, \Theta, \Gamma)$ contraction; $b$-metric-like; Fixed point

\section{Introduction and preliminaries}

Fixed point theory is still a central topic with a broad focus on applications of fixed point models not only in mathematical analysis, but also in other branches of natural sciences. The Banach contraction theorem continues to be generalized in other metric settings. For more related results, see [1-23].

Following the generalizations made by Matthews [24], Hitzler and Seda [25], and AminiHarandi [26], Alghamdi et al. [27] introduced the concept of $b$-metric-like spaces. Many authors have obtained interesting results in these areas associated with many more applications in the field of nonlinear analysis and main areas of interdisciplinary research.

In our work, we use the notions of $\alpha$-admissible functions, $(\alpha, \psi, \varphi)$-contractive mappings, $F$-contractions, and Kannan type contractions. In this paper, we introduce $\alpha_{s p}-$ $F$ contractive mappings by means of $\alpha_{s} p$-admissible functions and auxiliary functions, named as $C$-class functions. We also provide two wide classes of contractions selected among $b$-metric and $b$-metric-like settings, giving knew extensions of $\alpha_{s^{p}}-F$ contractions and Kannan type contractions. These new generalized classes not only generalize the known ones, but also include and unify a huge number of existing ones selected in the corresponding literature, and the corresponding results are supported by an application on boundary value problems.

Let $T$ be a nonempty set and $s \geq 1$ be a given real number. Let $\sigma_{b}: T \times T \rightarrow[0, \infty)$ be a mapping satisfying the following conditions for each $h, k, z \in T$ :

(c) The Author(s) 2021. This article is licensed under a Creative Commons Attribution 4.0 International License, which permits use, sharing, adaptation, distribution and reproduction in any medium or format, as long as you give appropriate credit to the original author(s) and the source, provide a link to the Creative Commons licence, and indicate if changes were made. The images or other third party material in this article are included in the article's Creative Commons licence, unless indicated otherwise in a credit line to the material. If material is not included in the article's Creative Commons licence and your intended use is not permitted by statutory regulation or exceeds the permitted use, you will need to obtain permission directly from the copyright holder. To view a copy of this licence, visit http://creativecommons.org/licenses/by/4.0/. 
I. $\sigma_{b}(h, k)=0$ if and only if $h=k$;

II. $\sigma_{b}(h, k)=0$ implies $h=k$;

III. $\sigma_{b}(h, k)=\sigma_{b}(k, h)$;

IV. $\sigma_{b}(h, k) \leq \sigma_{b}(h, z)+\sigma_{b}(z, k)$;

V. $\sigma_{b}(h, k) \leq s\left[\sigma_{b}(h, z)+\sigma_{b}(z, k)\right]$.

Definition 1.1 ([28]) A pair $\left(T, \sigma_{b}\right)$ satisfying axioms I, III, and V is called a $b$-metric space with parameter $s$.

Definition 1.2 ([26]) A pair $\left(T, \sigma_{b}\right)$ satisfying axioms II, III, and IV is called a metric-like space.

Definition 1.3 ([27]) A pair $\left(T, \sigma_{b}\right)$ satisfying axioms II, III, and V is called a $b$-metric-like space with parameter $s$.

It is true that if $h, k \in T$ and $\sigma_{b}(h, k)=0$, then $h=k$; however, the converse need not be true, and $\sigma_{b}(h, h)$ may be positive for $h \in T$.

Example 1.4 Let $T=\mathbb{R}$ and $\sigma_{b}: T^{2} \rightarrow[0, \infty)$ be a given function as $\sigma_{b}(h, k)=(|h|+|k|)^{2}$ for all $h, k \in T$. Then $\left(T, \sigma_{b}\right)$ is a $b$-metric-like space with parameter $s=2$.

Definition 1.5 ([27]) Let $\left(T, \sigma_{b}\right)$ be a $b$-metric-like space.

(a) A sequence $\left\{h_{n}\right\}$ in $T$ is called convergent to a point $h \in T$ if $\lim _{n \rightarrow \infty} \sigma_{b}\left(h_{n}, h\right)=\sigma_{b}(h, h)$;

(b) A sequence $\left\{h_{n}\right\}$ in $T$ is called Cauchy if $\lim _{n, m \rightarrow \infty} \sigma_{b}\left(h_{n}, h_{m}\right)$ exists and is finite;

(c) The $b$-metric-like space $\left(T, \sigma_{b}\right)$ is called complete if, for every Cauchy sequence $\left\{h_{n}\right\}$ in $T$, there exists $h \in T$ such that $\lim _{n, m \rightarrow \infty} \sigma_{b}\left(h_{n}, h_{m}\right)=\lim _{n \rightarrow \infty} \sigma_{b}\left(h_{n}, h\right)=\sigma_{b}(h, h)$.

In 2012, the introduction of $\alpha$-admissible functions by Samet et al. in [29] leads to an extensive development of many notions and properties related to fixed point theory and its applications.

Definition 1.6 Let $T$ be a nonempty set. Let $f: T \rightarrow T$ and $\alpha: T \times T \rightarrow \mathbb{R}^{+}$be given functions. We say that $f$ is an $\alpha$-admissible mapping if $\alpha(h, k) \geq 1$ implies that $\alpha(f h, f k) \geq 1$ for all $h, k \in T$.

Further, Aydi [30] extended this definition to a pair of mappings.

Definition 1.7 For a nonempty set $T$, let $f, g: T \rightarrow T$ and $\alpha: T \times T \rightarrow \mathbb{R}^{+}$be mappings. We say that $(f, g)$ is an $\alpha$-admissible pair if, for all $h, k \in T$ we have

$$
\alpha(h, k) \geq 1 \Rightarrow \alpha(f h, g k) \geq 1 \quad \text { and } \quad \alpha(g k, f h) \geq 1 .
$$

We here summarize the most important lemmas and results very useful in the main section of the paper. 
Lemma 1.8 ([21]) Let $\left(T, \sigma_{b}\right)$ be a b-metric-like space with parameter $s \geq 1$. If a given mapping $f: T \rightarrow T$ is continuous at $h^{*} \in T$, then we have

$$
\sigma_{b}\left(f h_{n}, f h^{*}\right) \rightarrow \sigma_{b}\left(f h^{*}, f h^{*}\right) \quad \text { whenever } \sigma_{b}\left(h_{n}, h^{*}\right) \rightarrow \sigma_{b}\left(h^{*}, h^{*}\right) \text { for each }\left\{h_{n}\right\} \text { in } T \text {. }
$$

The following is a short revised version of the lemma in [22].

Lemma 1.9 Let $\left(T, \sigma_{b}\right)$ be a b-metric-like space with parameter $s \geq 1$, and suppose that $\left\{h_{n}\right\}$ is $\sigma_{b}$-convergent to $h$ with $\sigma_{b}(h, h)=0$. Then, for each $j \in T$, we have

$$
s^{-1} \sigma_{b}(h, j) \leq \liminf _{n \rightarrow \infty} \sigma_{b}\left(h_{n}, j\right) \leq \limsup _{n \rightarrow \infty} \sigma_{b}\left(h_{n}, j\right) \leq s \sigma_{b}(h, j)
$$

Lemma 1.10 ([21]) In a b-metric-like space $\left(T, \sigma_{b}\right)$ with parameter $s \geq 1$, for $h, k \in T$ and $\left\{h_{n}\right\} \subset T$, we have:

(a) $\sigma_{b}(h, k)=0 \Rightarrow \sigma_{b}(h, h)=\sigma_{b}(k, k)=0$;

(b) If $\lim _{n \rightarrow \infty} \sigma_{b}\left(h_{n}, h_{n+1}\right)=0$, then $\lim _{n \rightarrow \infty} \sigma_{b}\left(h_{n}, h_{n}\right)=\lim _{n \rightarrow \infty} \sigma_{b}\left(h_{n+1}, h_{n+1}\right)=0$;

(c) $h \neq k \Rightarrow \sigma_{b}(h, k)>0$.

Lemma $1.11([22])$ Let $\left(T, \sigma_{b}\right)$ be a complete b-metric-like space and $\left\{h_{n}\right\}$ be a sequence such that

$$
\lim _{n \rightarrow \infty} \sigma_{b}\left(h_{n}, h_{n+1}\right)=0
$$

If, for such a sequence $\left\{h_{n}\right\}, \lim _{n, m \rightarrow \infty} \sigma_{b}\left(h_{n}, h_{m}\right) \neq 0$, then there are $\varepsilon>0$ and subsequences of positive integers $\{m(i)\} ;\{n(i)\}$ with $n_{i}>m_{i}>i$ such that

$$
\begin{aligned}
& \varepsilon \leq \lim \sup _{i \rightarrow \infty} \sigma_{b}\left(h_{2 n_{i}}, h_{2 m_{i}}\right) \leq \varepsilon s, \quad \varepsilon / s \leq \lim \sup _{i \rightarrow \infty} \sigma_{b}\left(h_{2 m_{i}}, h_{2 n_{i}-1}\right) \leq \varepsilon s, \\
& \varepsilon / s^{2} \leq \limsup _{i \rightarrow \infty} \sigma_{b}\left(h_{2 n_{i}-1}, h_{2 m_{i}+1}\right) \leq \varepsilon s^{2}, \\
& \varepsilon / s \leq \lim \sup _{i \rightarrow \infty} \sigma_{b}\left(h_{2 m_{i}+1}, h_{2 n_{i}}\right) \leq \varepsilon s^{2} .
\end{aligned}
$$

Lemma 1.12 ([22]) Let $\left\{h_{n}\right\}$ be a sequence in a b-metric-like space $\left(T, \sigma_{b}\right)$ with parameter $s \geq 1$ such that $\sigma_{b}\left(h_{n}, h_{n+1}\right) \leq \lambda \sigma_{b}\left(h_{n-1}, h_{n}\right)$ for all $n>0$, for some $\lambda$, where $0 \leq \lambda<1 / s$. Then:

1. $\lim _{n \rightarrow \infty} \sigma_{b}\left(h_{n}, h_{n+1}\right)=0$,

2. $\left\{h_{n}\right\}$ is a Cauchy sequence in $\left(T, \sigma_{b}\right)$ and $\lim _{n, m \rightarrow \infty} \sigma_{b}\left(h_{n}, h_{m}\right)=0$.

Definition 1.13 ([22]) Let $\left(T, \sigma_{b}\right)$ be a $b$-metric-like space, $f, g: T \rightarrow T$ and $\alpha: T \times T \rightarrow$ $\mathbb{R}^{+}$be given mappings, and let $p \geq 1$ be an arbitrary constant. We say that $(f, g)$ is an $\alpha_{s^{p}}$ admissible pair if $\alpha(h, k) \geq s^{p}$ implies $\min \{\alpha(f h, g k), \alpha(g k, f h)\} \geq s^{p}$ for all $h, k \in T$.

Examples 2 and 3 in [22] illustrate Definition 1.13.

Definition 1.14 ([22]) Let $\left(T, \sigma_{b}\right)$ be a $b$-metric-like space, $f: T \rightarrow T$ and $\alpha: T \times T \rightarrow$ $\mathbb{R}^{+}$be given mappings, and let $p \geq 1$ be an arbitrary constant. We say that $f$ is an $\alpha_{s^{p}}$ admissible mapping if $\alpha(h, k) \geq s^{p}$ implies $\min \{\alpha(f h, f k), \alpha(f k, f h)\} \geq s^{p}$ for all $h, k \in T$. 
Also, in the sequel, we recall additional properties given in [22].

$\left(H_{s^{p}}\right)$ : If $\left\{h_{n}\right\}$ is a sequence in $T$ such that $h_{n} \rightarrow h \in T$ as $n \rightarrow \infty$ and $\alpha\left(h_{n}, h_{n+1}\right) \geq s^{p}$ and $\alpha\left(h_{n+1}, h_{n}\right) \geq s^{p}$, then there exists a subsequence $\left\{h_{n_{i}}\right\}$ of $\left\{h_{n}\right\}$ with $\alpha\left(h_{n_{i}}, h\right) \geq s^{p}$ and $\alpha\left(h, h_{n_{i}}\right) \geq s^{p}$ for all $i \in \mathbb{N}$.

$\left(U_{s^{p}}\right)$ : For all $h, k \in C F(f, g)$, we have $\alpha(h, k) \geq s^{p}$, where $C F(f, g)$ denotes the set of common fixed points of $f$ and $g$ (also $\operatorname{Fix}(f)$ is the set of fixed points of $f$ ).

Definition 1.15 ([31]) A mapping $F: \mathbb{R}^{+} \times \mathbb{R}^{+} \rightarrow \mathbb{R}$ is called a $C$-class function if

1) $F(m, n) \leq m$ for all $m, n \geq 0$;

2) $F(m, n)=m$ implies that either $m=0$ or $n=0$ for all $m, n \geq 0$;

3) $F(m, n)$ is continuous on its variables $m, n \geq 0$.

\section{Main results}

In this section we present two main theorems. The first is a general result in a larger ambient of spaces that extends and unifies a number of well-known corresponding results related to fixed point theory. The second is an extension of the outstanding classical result of Kannan contraction to the setting of $b$-metric-like spaces.

Let $f: T \rightarrow T$ be a mapping. We denote

$$
N(h, k)=\max \left\{\begin{array}{l}
\sigma_{b}(h, k), \sigma_{b}(h, f h), \sigma_{b}(k, f k), \frac{\sigma_{b}(h, f k)+\sigma_{b}(k, f h)}{4 s}, \frac{\sigma_{b}(h, f h) \sigma_{b}(h, f k)}{1+s\left[\sigma_{b}(h, f h)+\sigma_{b}(k, f k)\right]}, \\
\frac{\sigma_{b}(k, f k)\left[1+\sigma_{b}(h, f h)\right]}{1+\sigma_{b}(h, k)}, \frac{\sigma_{b}(h, f h)\left[1+\sigma_{b}(k, f k)\right]}{1+\sigma_{b}(f h, f k)}
\end{array}\right\}
$$

and the following sets of functions:

$$
\begin{aligned}
\mathrm{I}:= & \{\psi:[0,+\infty) \rightarrow[0,+\infty) \text { is strictly increasing, continuous such that } \psi(m)=0 \\
& \text { iff } m=0\} \\
\Theta: & :\{\theta:[0,+\infty) \rightarrow[0,+\infty) \text { is continuous with } \theta(m)<\psi(m) \text { for all } m>0\} ; \\
\Gamma:= & \left\{\begin{array}{l}
(\beta, \gamma, \delta) / \beta, \gamma, \delta: R^{+} \rightarrow[0,1) \text { satisfying } \\
\lim \sup _{n \rightarrow m} \beta(n)+\lim \sup _{n \rightarrow m} \gamma(n)+\lim \sup _{n \rightarrow m} \delta(n)<1, \text { for all } m>0
\end{array}\right\} .
\end{aligned}
$$

Definition 2.1 Let $\left(T, \sigma_{b}\right)$ be a $b$-metric-like space with parameter $s \geq 1$. A mapping $f$ : $T \rightarrow T$ is said to be an $\alpha_{s^{p}}-(C, N, \Theta)$ contraction if $f$ is an $\alpha_{s^{p}}$-admissible mapping $(p>1)$ and it satisfies

$$
\alpha(h, k) \sigma_{b}(f h, f k) \leq F(N(h, k), \theta(N(h, k)))
$$

for all $h, k \in T$, where $F \in C, \theta \in \Theta$ and $N(h, k)$ is defined by (1).

We now state the following general result.

Theorem 2.2 Let $\left(T, \sigma_{b}\right)$ be a complete b-metric-like space with parameter $s>1$, and $f$ : $T \rightarrow T$ be an $\alpha_{s}-(C, N, \Theta)$ contraction. Suppose that the following conditions hold:

(i) there exists $h_{0} \in T$ such that $\min \left\{\alpha\left(h_{0}, f h_{0}\right), \alpha\left(f h_{0}, h_{0}\right)\right\} \geq s^{p}$;

(ii) the properties $H_{s^{p}}$ and $U_{s^{p}}$ are satisfied.

Then $f$ has a unique fixed point $h \in T$. 
Proof From assumption (i) there exists $h_{0} \in T$ such that $\alpha\left(h_{0}, f h_{0}\right) \geq s^{p}$. We construct a sequence $\left\{h_{n}\right\}$ in $T$ by $h_{n}=f^{n} h_{0}=f\left(h_{n-1}\right)$ for all $n \in \mathbb{N}$. If we suppose that $\sigma_{b}\left(h_{n}, h_{n+1}\right)=0$ for some $n$, then $h_{n+1}=h_{n}$, and so $f$ has a fixed point. Consequently, throughout the proof, we assume that

$$
\sigma_{b}\left(h_{n}, h_{n+1}\right)>0 \text { for all } n \in \mathbb{N} \text {. }
$$

By the $\alpha_{s} p$-type admissibility of $f$, we observe that

$$
\begin{aligned}
& \alpha\left(h_{0}, h_{1}\right)=\alpha\left(h_{0}, f h_{0}\right) \geq s^{p}, \\
& \alpha\left(f h_{0}, f h_{1}\right)=\alpha\left(h_{1}, h_{2}\right) \geq s^{p} \quad \text { and } \quad \alpha\left(f h_{1}, f h_{2}\right)=\alpha\left(h_{2}, h_{3}\right) \geq s^{p} .
\end{aligned}
$$

Then, inductively,

$$
\alpha\left(h_{n}, h_{n+1}\right) \geq s^{p} \quad \text { for all } n \in \mathbb{N} \text {. }
$$

By (1) and condition (2), we have

$$
\begin{aligned}
s^{p} \sigma_{b}\left(h_{n}, h_{n+1}\right) & =s^{p} \sigma_{b}\left(f h_{n-1}, f h_{n}\right) \\
& \leq \alpha\left(h_{n-1}, h_{n}\right) \sigma_{b}\left(f h_{n-1}, f h_{n}\right) \\
& \leq F\left(N\left(h_{n-1}, h_{n}\right), \theta\left(N\left(h_{n-1}, h_{n}\right)\right)\right) \\
& \leq N\left(h_{n-1}, h_{n}\right),
\end{aligned}
$$

where

$$
\begin{aligned}
N\left(h_{n-1}, h_{n}\right)= & \max \left\{\begin{array}{l}
\sigma_{b}\left(h_{n-1}, h_{n}\right), \sigma_{b}\left(h_{n-1}, f h_{n-1}\right), \sigma_{b}\left(h_{n}, f h_{n}\right), \frac{\sigma_{b}\left(h_{n-1} f h_{n}\right)+\sigma_{b}\left(h_{n} f h_{n-1}\right)}{4 s}, \\
\frac{\sigma_{b}\left(h_{n-1}, h_{n}\right) \sigma_{b}\left(h_{n-1} f h_{n}\right)}{1+s\left[\sigma_{b}\left(h_{n-1} f h_{n-1}\right)+\sigma_{b}\left(h_{n}, f h_{n}\right)\right]} \frac{\sigma_{b}\left(h_{n}, f h_{n}\right)\left[1+\sigma_{b}\left(h_{n-1} f h_{n-1}\right)\right]}{1+\sigma_{b}\left(h_{n-1}, h_{n}\right)}, \\
\frac{\sigma_{b}\left(h_{n-1} f h_{n-1}\right)\left[1+\sigma_{b}\left(h_{n}, f h_{n}\right)\right]}{1+\sigma_{b}\left(f h_{n-1} f h_{n}\right)}
\end{array}\right\} \\
= & \max \left\{\begin{array}{l}
\sigma_{b}\left(h_{n-1}, h_{n}\right), \sigma_{b}\left(h_{n-1}, h_{n}\right), \sigma_{b}\left(h_{n}, h_{n+1}\right), \frac{\sigma_{b}\left(h_{n-1}, h_{n+1}\right)+\sigma_{b}\left(h_{n}, h_{n}\right)}{4}, \\
\frac{\sigma_{b}\left(h_{n-1}, h_{n}\right) \sigma_{b}\left(h_{n-1}, h_{n+1}\right)}{1+s\left[\sigma_{b}\left(h_{n-1}, h_{n}\right)+\sigma_{b}\left(h_{n}, h_{n+1}\right)\right]} \frac{\sigma_{b}\left(h_{n}, h_{n+1}\right)\left[1+\sigma_{b}\left(h_{n-1}, h_{n}\right)\right]}{1+\sigma_{b}\left(h_{n-1}, h_{n}\right)}, \\
\frac{\sigma_{b}\left(h_{n-1}, h_{n}\right)\left[1+\sigma_{b}\left(h_{n}, h_{n+1}\right)\right]}{1+\sigma_{b}\left(h_{n}, h_{n+1}\right)}
\end{array}\right\} \\
\leq & \max \left\{\begin{array}{l}
\sigma_{b}\left(h_{n-1}, h_{n}\right), \sigma_{b}\left(h_{n-1}, h_{n}\right), \sigma_{b}\left(h_{n}, h_{n+1}\right), \\
\frac{s\left[\sigma_{b}\left(h_{n-1}, h_{n}\right)+\sigma_{b}\left(h_{n}, h_{n+1}\right)\right]+2 s \sigma_{b}\left(h_{n-1}, h_{n}\right)}{4 s}, \\
\frac{\sigma_{b}\left(h_{n-1}, h_{n}\right) s\left[\sigma_{b}\left(h_{n-1}, h_{n}\right)+\sigma_{b}\left(h_{n}, h_{n+1}\right)\right]}{1+s\left[\sigma_{b}\left(h_{n-1}, h_{n}\right)+\sigma_{b}\left(h_{n}, h_{n+1}\right)\right]}, \sigma_{b}\left(h_{n}, h_{n+1}\right), \sigma_{b}\left(h_{n-1}, h_{n}\right)
\end{array}\right\} \\
= & \max \left\{\begin{array}{l}
\left.\sigma_{b}\left(h_{n-1}, h_{n}\right), \sigma_{b}\left(h_{n}, h_{n+1}\right)\right\} .
\end{array}\right.
\end{aligned}
$$

If we have

$$
\sigma_{b}\left(h_{n-1}, h_{n}\right)<\sigma_{b}\left(h_{n}, h_{n+1}\right) \text { for some } n \in \mathbb{N} \text {, }
$$

then, from inequality (6), we get

$$
N\left(h_{n-1}, h_{n}\right) \leq \sigma_{b}\left(h_{n}, h_{n+1}\right) .
$$


Using (5), we obtain

$$
s^{p} \sigma_{b}\left(h_{n}, h_{n+1}\right) \leq \sigma_{b}\left(h_{n}, h_{n+1}\right) .
$$

Hence $\sigma_{b}\left(h_{n}, h_{n+1}\right)=0$, that is a contradiction.

Thus, for all $n \in \mathbb{N}, \sigma_{b}\left(h_{n}, h_{n+1}\right) \leq \sigma_{b}\left(h_{n-1}, h_{n}\right)$ and by (5), we can establish that

$$
s^{p} \sigma_{b}\left(h_{n}, h_{n+1}\right) \leq \sigma_{b}\left(h_{n-1}, h_{n}\right) .
$$

As a result, the above inequality can be written as

$$
\sigma_{b}\left(h_{n}, h_{n+1}\right) \leq \lambda \sigma_{b}\left(h_{n-1}, h_{n}\right)
$$

where $\lambda=1 / s^{p} \in[0,1 / s)$. By Lemma 1.12 and using (8), we claim

$$
\lim _{n \rightarrow \infty} \sigma_{b}\left(h_{n}, h_{n+1}\right)=0
$$

and the sequence $\left\{h_{n}\right\}$ is Cauchy. $\left(T, \sigma_{b}\right)$ is complete, so there is some $h \in T$ such that $\left\{h_{n}\right\}$ converges to $h$. That is,

$$
\lim _{n \rightarrow \infty} \sigma_{b}\left(h_{n}, h\right)=\sigma_{b}(h, h)=\lim _{n, m \rightarrow \infty} \sigma_{b}\left(h_{n}, h_{m}\right)=0 .
$$

The self-map $f$ is not continuous, then from (4) and property $H_{s} p$, there exists a subsequence $\left\{h_{n_{i}}\right\}$ of $\left\{h_{n}\right\}$ such that $\alpha\left(h_{n_{i}}, h\right) \geq s^{p}$ for all $i \in \mathbb{N}$. Applying contractive condition (2) to $h_{n_{i}}$ and $h$, we obtain

$$
\begin{aligned}
s^{p} \sigma_{b}\left(h_{n_{i}+1}, f h\right) & =s^{p} \sigma_{b}\left(f h_{n_{i}}, f h\right) \\
& \leq \alpha\left(h_{n_{i}}, h\right) \sigma_{b}\left(f h_{n_{i}}, f h\right) \\
& \leq F\left(N\left(h_{n_{i}}, h\right), \theta\left(N\left(h_{n_{i}}, h\right)\right)\right) \\
& \leq N\left(h_{n_{i}}, h\right),
\end{aligned}
$$

where

$$
\begin{aligned}
N\left(h_{n_{i}}, h\right) & =\max \left\{\begin{array}{l}
\sigma_{b}\left(h_{n_{i}}, h\right), \sigma_{b}\left(h_{n_{i}}, f h_{n_{i}}\right), \sigma_{b}(h, f h), \\
\frac{\sigma_{b}\left(h_{n_{i}} f h\right)+\sigma_{b}\left(h_{i} f h_{n_{i}}\right)}{4 s}, \frac{\sigma_{b}\left(h_{n_{i}}, h\right) \sigma_{b}\left(h_{n_{i}} f h\right)}{1+s\left[\sigma_{b}\left(h_{n_{i}}, f h_{n_{i}}\right)+\sigma_{b}(h, f h)\right]} \\
\frac{\sigma_{b}\left(h_{f} f h\right)\left[1+\sigma_{b}\left(h_{n_{i}} f h_{n_{i}}\right)\right]}{1+\sigma_{b}\left(h_{n_{i}}, h\right)}, \frac{\sigma_{b}\left(h_{n_{i}} f h_{n_{i}}\right)\left[1+\sigma_{b}(h, f h)\right]}{1+\sigma_{b}\left(f h_{n_{i}} f h\right)}
\end{array}\right\} \\
= & \max \left\{\begin{array}{l}
\sigma_{b}\left(h_{n_{i}}, h\right), \sigma_{b}\left(h_{n_{i}}, h_{n_{i}+1}\right), \sigma_{b}(h, f h), \\
\frac{\sigma_{b}\left(h_{n_{i}} f h\right)+\sigma_{b}\left(h, h_{n_{i}+1}\right)}{4 s}, \frac{\sigma_{b}\left(h_{n_{i}}, h\right) \sigma_{b}\left(h_{n_{i}}, f h\right)}{1+s\left[\sigma_{b}\left(h_{n_{i}}, h_{n_{i}+1}\right)+\sigma_{b}(h, f h)\right]} \\
\frac{\sigma_{b}(h, f h)\left[1+\sigma_{b}\left(h_{n_{i}}, h_{n_{i}+1}\right)\right]}{1+\sigma_{b}\left(h_{n_{i}}, h\right)}, \frac{\sigma_{b}\left(h_{n_{i}}, h_{n_{i}+1}\right)\left[1+\sigma_{b}(h, f h)\right]}{1+\sigma_{b}\left(h_{n_{i}+1} f h\right)}
\end{array}\right\} .
\end{aligned}
$$

By the upper limit in (12) and due to Lemma 1.9, Lemma 1.10, and Eq. (10), we derive

$$
\limsup _{i \rightarrow \infty} N\left(h_{n_{i}}, h\right) \leq \max \left\{0,0, \sigma_{b}(h, f h), \frac{s \sigma_{b}(h, f h)}{4 s}, 0, \sigma_{b}(h, f h), 0\right\}=\sigma_{b}(h, f h) .
$$


Letting $i \rightarrow \infty$ in (11), and in view of (13) and Lemma 1.9, it follows that

$$
\begin{aligned}
s^{p-1} \sigma_{b}(h, f h) & =s^{p} \frac{1}{s} \sigma_{b}(h, f h) \leq s^{p} \lim \sup _{i \rightarrow \infty} \sigma_{b}\left(h_{n_{i}}, f h\right) \\
& \leq \lim \sup _{i \rightarrow \infty} N\left(h_{n_{i}}, h\right) \leq \sigma_{b}(h, f h) .
\end{aligned}
$$

From (14) we get $\sigma_{b}(h, f h)=0$, which implies that $f h=h$. Hence $h$ is a fixed point of $f$.

If $h, z \in \operatorname{Fix}(f)$, by the hypothesis $U_{s}, \alpha(h, z) \geq s^{p}$, and applying (2), we have

$$
\begin{aligned}
s^{p} \sigma_{b}(h, h) & =s^{p} \sigma_{b}(f h, f h) \leq \alpha(h, h) \sigma_{b}(f h, f h) \\
& \leq F(N(h, h), \theta(N(h, h))) \\
& \leq N(h, h)=\sigma_{b}(h, h),
\end{aligned}
$$

where

$$
\begin{aligned}
N(h, h) & =\max \left\{\begin{array}{l}
\sigma_{b}(h, h), \sigma_{b}(h, f h), \sigma_{b}(h, f h), \frac{\sigma_{b}(h, f h)+\sigma_{b}(h, f h)}{4 s}, \frac{\sigma_{b}(h, f h) \sigma_{b}(h, f h)}{1+s\left[\sigma_{b}(h, f h)+\sigma_{b}(h, f h)\right]}, \\
\frac{\sigma_{b}(h, f h)\left[1+\sigma_{b}(h, f h)\right]}{1+\sigma_{b}(h, h)}, \frac{\sigma_{b}(h, f h)\left[1+\sigma_{b}(h, f h)\right]}{1+\sigma_{b}(f h, f h)}
\end{array}\right\} \\
& =\max \left\{\begin{array}{l}
\sigma_{b}(h, h), \sigma_{b}(h, h), \sigma_{b}(h, h), \frac{\sigma_{b}(h, h)+\sigma_{b}(h, h)}{4 s}, \frac{\sigma_{b}(h, h) \sigma_{b}(h, h)}{1+s\left[\sigma_{b}(h, h)+\sigma_{b}(h, h)\right]}, \\
\frac{\sigma_{b}(h, h)\left[1+\sigma_{b}(h, h)\right]}{1+\sigma_{b}(h, h)}, \frac{\sigma_{b}(h, h)\left[1+\sigma_{b}(h, h)\right]}{1+\sigma_{b}(h, h)}
\end{array}\right\} \\
& =\sigma_{b}(h, h) .
\end{aligned}
$$

By (15) it follows $s^{p} \sigma_{b}(h, h) \leq \sigma_{b}(h, h)$.

Since $s>1$, the inequality above implies $\sigma_{b}(h, h)=0\left(\operatorname{similarly,} \sigma_{b}(z, z)=0\right)$.

Again by condition (2), we have

$$
\begin{aligned}
s^{p} \sigma_{b}(h, z) & =s^{p} \sigma_{b}(f h, f z) \leq \alpha(h, z) \sigma_{b}(f h, f z) \\
& \leq F(N(h, z), \theta(N(h, z))) \\
& \leq N(h, z) \\
& =\sigma_{b}(h, z),
\end{aligned}
$$

where

$$
\begin{aligned}
& N(h, z)=\max \left\{\begin{array}{l}
\sigma_{b}(h, z), \sigma_{b}(h, f h), \sigma_{b}(z, f z), \frac{\sigma_{b}(h, f z)+\sigma_{b}(z, f h)}{4 s}, \frac{\sigma_{b}(h, f h) \sigma_{b}(h, f z)}{1+s\left[\sigma_{b}(h, f h)+\sigma_{b}(z, f z)\right]}, \\
\frac{\sigma_{b}(z, f z)\left[1+\sigma_{b}(h, f h)\right]}{1+\sigma_{b}(h, z)}, \frac{\sigma_{b}(h, f h)\left[1+\sigma_{b}(z, f z)\right]}{1+\sigma_{b}(f h, f z)}
\end{array}\right\} \\
& =\max \left\{\begin{array}{l}
\sigma_{b}(h, z), \sigma_{b}(h, h), \sigma_{b}(z, z), \frac{\sigma_{b}(h, z)+\sigma_{b}(z, h)}{4 s}, \frac{\sigma_{b}(h, h) \sigma_{b}(h, z)}{1+s\left[\sigma_{b}(h, h)+\sigma_{b}(z, z)\right]}, \\
\frac{\sigma_{b}(z, z)\left[1+\sigma_{b}(h, h)\right]}{1+\sigma_{b}(h, z)}, \frac{\sigma_{b}(h, h)\left[1+\sigma_{b}(z, z)\right]}{1+\sigma_{b}(h, z)}
\end{array}\right\} \\
& =\max \left\{\sigma_{b}(h, z), 0,0, \frac{\sigma_{b}(h, z)}{2 s}, 0,0,0\right\} \\
& =\sigma_{b}(h, z) \text {. }
\end{aligned}
$$

Inequality (18) implies that $\sigma_{b}(h, z)=0$. Therefore, $h=z$ and the fixed point is unique. 


\section{Remark 2.3}

(i) The proof of Theorem 2.2 is simply constructive somewhat shorter, and avoid the use of Lemma 1.11.

(ii) The above result reduces to other settings of spaces for the choice of parameters $s$ and $p$.

(iii) Many applications of Theorem 2.2 are attributed to the variety of class $C$ that makes it to contain many known theorems as special cases.

In the sequel, we provide an illustrative example of Theorem 2.2.

Example 2.4 In $T=[0,+\infty)$, we take $\sigma_{b}(h, k)=(h+k)^{2}$ for all $h, k \in T$. Clearly, $\left(T, \sigma_{b}\right)$ is a $b$-metric-like space with coefficient $s=2$. Let us define the mappings $f: T \rightarrow T$ and $\alpha: T \times T \rightarrow[0,+\infty[$ by

$$
f h=\left\{\begin{array}{ll}
\frac{1}{5} h & \text { if } h \in[0,1), \\
\frac{1}{10} h & \text { if } h \in[1,2), \\
2 h & \text { if } h \geq 2
\end{array} \quad \text { and } \quad \alpha(h, k)= \begin{cases}h+k+4 & \text { if } h, k \in[0,2] \\
0 & \text { otherwise }\end{cases}\right.
$$

Let $h, k \in T$, if $\alpha(h, k) \geq 4=s^{2}$, then $h, k \in[0,2)$, and also we have $f h, f k \in[0,1 / 5)$ and $\alpha(f h, f k) \geq s^{2}$. Thus we have shown that $f$ is an $\alpha_{s} p$-admissible mapping. Choosing $F \in C$ as $\mathrm{F}(m, n)=m-n$, we discuss the following cases:

Let $h, k \in[0,1)$, then we get

$$
\begin{aligned}
\alpha(h, k) \sigma_{b}(f h, f k) & =s^{2} \sigma_{b}(f h, f k)=4 \sigma_{b}\left(\frac{1}{5} h, \frac{1}{5} k\right)=4\left(\frac{1}{5} h+\frac{1}{5} k\right)^{2} \\
& =\frac{4}{25}(h+k)^{2}<\frac{1}{6} \sigma_{b}(h, k) \leq \frac{1}{6} N(h, k)=N(h, k)-\frac{5}{6} N(h, k) \\
& =N(h, k)-\theta(N(h, k)) \\
& =F(N(h, k), \theta(N(h, k))) .
\end{aligned}
$$

Let $h, k \in[1,2)$, then we get

$$
\begin{aligned}
\alpha(h, k) \sigma_{b}(f h, f k) & =s^{2} \sigma_{b}(f h, f k)=4 \sigma_{b}\left(\frac{1}{10} h, \frac{1}{10} k\right)=4\left(\frac{1}{10} h+\frac{1}{10} k\right)^{2} \\
& =\frac{4}{100}(h+k)^{2}<\frac{1}{6} \sigma_{b}(h, k) \leq \frac{1}{6} N(h, k)=N(h, k)-\frac{5}{6} N(h, k) \\
& =N(h, k)-\theta(N(h, k)) \\
& =F(N(h, k), \theta(N(h, k))) .
\end{aligned}
$$

Let $h \in[0,1), k \in[1,2)$, then we get

$$
\begin{aligned}
\alpha(h, k) \sigma_{b}(f h, f k) & =s^{2} \sigma_{b}(f h, f k)=4 \sigma_{b}\left(\frac{1}{5} h, \frac{1}{10} k\right)=4\left(\frac{1}{5} h+\frac{1}{10} k\right)^{2} \leq 4\left(\frac{1}{5} h+\frac{1}{5} k\right)^{2} \\
& =\frac{4}{25}(h+k)^{2}<\frac{1}{6} \sigma_{b}(h, k) \leq \frac{1}{6} N(h, k)=N(h, k)-\frac{5}{6} N(h, k)
\end{aligned}
$$




$$
\begin{aligned}
& =N(h, k)-\theta(N(h, k)) \\
& =F(N(h, k), \theta(N(h, k))) .
\end{aligned}
$$

The other case $k \in[0,1), h \in[1,2)$ is the same as the previous case.

Obviously, the other assumptions of Theorem 2.2 can be verified and $f$ has $h=0$ as a unique fixed point.

On the other hand, if we refer to the metric space with the standard metric $d(h, k)=$ $|h-k|$ for points $h=0, k=2$ in case $N(0,2)=d(0,2)$, we see that

$$
4=d(f 0, f 2) \leq F(d(0,2), \theta(d(0,2)))=F(2, \theta(2)),
$$

that is, there exists no function $F \in C$ that satisfies the inequality (and also the Banach contraction principle).

Theorem 2.5 Let $\left(T, \sigma_{b}\right)$ be a complete $b$-metric-like space with parameter $s>1$ and $f$ : $T \rightarrow T$ be a mapping satisfying

$$
s^{p} \sigma_{b}(f h, f k) \leq F(N(h, k), \theta(N(h, k)))
$$

for all $h, k \in T$, where $\theta \in \Theta, F \in C, p>1$ and $N(h, k)$ is defined by (1). Then $f$ has a fixed point in $T$.

Proof It is obtained from Theorem 2.2 by setting $\alpha(h, k)=s^{p}(p>1)$.

Some applications of Theorem 2.2 are the following results by choosing the function $F \in C$, based on Example 2.13 (see [31]).

Corollary 2.6 Let $f: T \rightarrow T$ be an $\alpha_{s}$-admissible mapping on a complete b-metric-like space $\left(T, \sigma_{b}\right)$ with parameter $s>1$. Suppose that the following assertions hold:

(i) There exists a function $\beta:[0, \infty) \rightarrow[0,1)$ satisfying the condition: $\beta\left(h_{n}\right) \rightarrow 1$ as $n \rightarrow \infty$ implies that $h_{n} \rightarrow 0$ as $n \rightarrow \infty$ such that

$$
\alpha(h, k) \sigma_{b}(f h, f k) \leq \beta(N(h, k))(N(h, k))
$$

for all $h, k \in T$; where $N(h, k)$ is defined by $(1)$;

(ii) There exists $h_{0} \in T$ with $\min \left\{\alpha\left(h_{0}, f h_{0}\right), \alpha\left(f h_{0}, h_{0}\right)\right\} \geq s^{p}$;

(iii) Properties $H_{s}$; $U_{s}$ are satisfied.

Then $f$ has a unique fixed point $h \in T$.

Proof It follows from Theorem 2.2 by setting the function $F \in C$ as $F(m, n)=\beta(m) m$.

Corollary 2.7 Let $f: T \rightarrow T$ be an $\alpha_{s}$-admissible mapping on a complete b-metric-like space $\left(T, \sigma_{b}\right)$ with parameter $s>1$. Suppose that the following conditions are satisfied:

(i) There exists a continuous function $\varphi: \mathbb{R}^{+} \rightarrow \mathbb{R}^{+}$such that $\varphi(t)<t$ for all $t>0$, satisfying

$$
\alpha(h, k) \sigma_{b}(f h, f k) \leq \varphi(N(h, k))
$$


for all $h, k \in T$, where $N(h, k)$ is defined by (1);

(ii) There exists $h_{0} \in T$ with $\min \left\{\alpha\left(h_{0}, f h_{0}\right), \alpha\left(f h_{0}, h_{0}\right)\right\} \geq s^{p}$;

(iii) Properties $H_{s^{p}} ; U_{s^{p}}$ are satisfied.

Then $f$ has a unique fixed point $h \in T$.

Proof It is derived from Theorem 2.2 by setting $F(m, n)=\varphi(m)$ where $\varphi: \mathbb{R}^{+} \rightarrow \mathbb{R}^{+}$is continuous such that $\varphi(m)<m$ for all $m>0$.

The next theorem is a new extension of Kannan type contraction and is concerned with common fixed points for a pair of self-mappings. It uses the following definitions.

Definition 2.8 Let $\left(T, \sigma_{b}\right)$ be a complete $b$-metric-like space with parameter $s \geq 1$, and $f, g: T \rightarrow T, \alpha: T \times T \rightarrow \mathbb{R}^{+}$be given mappings. The pair $(f, g)$ is called a generalized $\alpha_{s^{p}}-(I, \Theta, \Gamma)$-Kannan contraction pair if there exist $\psi \in I, \theta \in \Theta, \beta, \gamma, \delta \in \Gamma$ satisfying

$$
\begin{aligned}
\psi\left(\alpha(h, k) \sigma_{b}(f h, g k)\right) \leq & \beta\left[\sigma_{b}(h, k)\right] \theta\left[\sigma_{b}(h, k)\right] \\
& +\gamma\left[\sigma_{b}(h, k)\right] \theta\left[\sigma_{b}(h, f h)\right] \\
& +\delta\left[\sigma_{b}(h, k)\right] \theta\left[\sigma_{b}(k, g k)\right]
\end{aligned}
$$

for all $h, k \in T$ with $\alpha(h, k) \geq s^{p}(p>1)$ and $\theta(m)<\psi(m)$ for all $m>0$.

Remark 2.9 If we put $g=f$, then Definition 2.8 can be stated as generalized $\alpha_{s} p-(I, \Theta, \Gamma)$ Kannan contraction for one mapping.

Theorem 2.10 Let $(f, g)$ be a pair of self-mappings on a complete b-metric-like space $\left(T, \sigma_{b}\right)$ with coefficient $s \geq 1$. If $(f, g)$ is a generalized $\alpha_{s} p-(I, \Theta, \Gamma)$ Kannan contraction pair, and the following conditions hold:

(i) There exists $h_{0} \in T$ with $\min \left\{\alpha\left(h_{0}, f h_{0}\right), \alpha\left(f h_{0}, h_{0}\right)\right\} \geq s^{p}$;

(ii) $(f, g)$ is an $\alpha_{s}$-admissible pair;

(iii) Properties $H_{s^{p}} ; U_{s^{p}}$ are satisfied.

Then $f$ and $g$ have a unique common fixed point $h \in T$.

Proof Since condition (i) holds, there exists $h_{0} \in T$ with $\alpha\left(h_{0}, f h_{0}\right) \geq s^{p}$ and $\alpha\left(f h_{0}, h_{0}\right) \geq s^{p}$. Take $h_{1}=f h_{0}$ and $h_{2}=g h_{1}$. By induction, we construct an iterative sequence $\left\{h_{n}\right\}$ in $T$ such that $h_{2 n+1}=f h_{2 n}$ and $h_{2 n+2}=g h_{2 n+1}$ for all $n \geq 0$. Then $\alpha\left(h_{0}, h_{1}\right) \geq s^{p}$ and $\alpha\left(h_{1}, h_{0}\right) \geq s^{p}$, by condition (ii) $(f, g)$ is an $\alpha_{s^{p}}$-admissible pair, so we obtain that

$$
\alpha\left(h_{1}, h_{2}\right)=\alpha\left(f h_{0}, g h_{1}\right) \geq s^{p} \quad \text { and } \quad \alpha\left(h_{2}, h_{1}\right)=\alpha\left(g h_{1}, f h_{0}\right) \geq s^{p} .
$$

Also, we have

$$
\alpha\left(h_{3}, h_{2}\right)=\alpha\left(f h_{2}, g h_{1}\right) \geq s^{p} \quad \text { and } \quad \alpha\left(h_{2}, h_{3}\right)=\alpha\left(g h_{1}, f h_{2}\right) \geq s^{p} .
$$

Proceeding inductively, we obtain

$$
\alpha\left(h_{n}, h_{n+1}\right) \geq s^{p} \quad \text { and } \quad \alpha\left(h_{n+1}, h_{n}\right) \geq s^{p} \quad \text { for all } n \geq 0 .
$$


If, for some $n \in N, \sigma_{b}\left(h_{2 n+1}, h_{2 n}\right)=0$, then by (19) we have

$$
\begin{aligned}
\psi\left(\sigma_{b}\left(h_{2 n+1}, h_{2 n+2}\right)\right) \leq & \psi\left(s^{p} \sigma_{b}\left(h_{2 n+1}, h_{2 n+2}\right)\right)=\psi\left(s^{p} \sigma_{b}\left(f h_{2 n}, g h_{2 n+1}\right)\right) \\
\leq & \psi\left(\alpha\left(h_{2 n}, h_{2 n+1}\right) \sigma_{b}\left(f h_{2 n}, g h_{2 n+1}\right)\right) \\
\leq & \beta\left[\sigma_{b}\left(h_{2 n}, h_{2 n+1}\right)\right] \theta\left[\sigma_{b}\left(h_{2 n}, h_{2 n+1}\right)\right] \\
& +\gamma\left[\sigma_{b}\left(h_{2 n}, h_{2 n+1}\right)\right] \theta\left[\sigma_{b}\left(h_{2 n}, f h_{2 n}\right)\right] \\
& +\delta\left[\sigma_{b}\left(h_{2 n}, h_{2 n+1}\right)\right] \theta\left[\sigma_{b}\left(h_{2 n+1}, g h_{2 n+1}\right)\right] \\
= & \beta\left[\sigma_{b}\left(h_{2 n}, h_{2 n+1}\right)\right] \theta\left[\sigma_{b}\left(h_{2 n}, h_{2 n+1}\right)\right] \\
& +\gamma\left[\sigma_{b}\left(h_{2 n}, h_{2 n+1}\right)\right] \theta\left[\sigma_{b}\left(h_{2 n}, h_{2 n+1}\right)\right] \\
& +\delta\left[\sigma_{b}\left(h_{2 n}, h_{2 n+1}\right)\right] \theta\left[\sigma_{b}\left(h_{2 n+1}, h_{2 n+2}\right)\right] \\
= & \delta\left[\sigma_{b}\left(h_{2 n}, h_{2 n+1}\right)\right] \theta\left[\sigma_{b}\left(h_{2 n+1}, h_{2 n+2}\right)\right]<\theta\left[\sigma_{b}\left(h_{2 n+1}, h_{2 n+2}\right)\right] .
\end{aligned}
$$

By properties of $\psi, \theta$, we get $\sigma_{b}\left(h_{2 n+1}, h_{2 n+2}\right)=0$, that is, $h_{2 n+1}=h_{2 n+2}$. Furthermore, that is $h_{2 n}=h_{2 n+1}=f h_{2 n}$ and $h_{2 n}=h_{2 n+2}=g h_{2 n+1}=g f h_{2 n}=g h_{2 n}$. Hence, the proof is concluded. Now, we assume that $\sigma_{b}\left(h_{n}, h_{n+1}\right)>0$ for all $n \geq 0$. By (20), applying condition (19), we have

$$
\begin{aligned}
\psi\left(\sigma_{b}\left(h_{2 n+1}, h_{2 n}\right)\right) \leq & \psi\left(s^{p} \sigma_{b}\left(h_{2 n+1}, h_{2 n}\right)\right)=\psi\left(s^{p} \sigma_{b}\left(f h_{2 n}, g h_{2 n-1}\right)\right) \\
\leq & \psi\left(\alpha\left(h_{2 n}, h_{2 n-1}\right) \sigma_{b}\left(f h_{2 n}, g h_{2 n-1}\right)\right) \\
\leq & \beta\left[\sigma_{b}\left(h_{2 n}, h_{2 n-1}\right)\right] \theta\left[\sigma_{b}\left(h_{2 n}, h_{2 n-1}\right)\right] \\
& +\gamma\left[\sigma_{b}\left(h_{2 n}, h_{2 n-1}\right)\right] \theta\left[\sigma_{b}\left(h_{2 n}, f h_{2 n}\right)\right] \\
& +\delta\left[\sigma_{b}\left(h_{2 n}, h_{2 n-1}\right)\right] \theta\left[\sigma_{b}\left(h_{2 n-1}, g h_{2 n-1}\right)\right] \\
= & \beta\left[\sigma_{b}\left(h_{2 n}, h_{2 n-1}\right)\right] \theta\left[\sigma_{b}\left(h_{2 n}, h_{2 n-1}\right)\right] \\
& +\gamma\left[\sigma_{b}\left(h_{2 n}, h_{2 n-1}\right)\right] \theta\left[\sigma_{b}\left(h_{2 n}, h_{2 n+1}\right)\right] \\
& +\delta\left[\sigma_{b}\left(h_{2 n}, h_{2 n-1}\right)\right] \theta\left[\sigma_{b}\left(h_{2 n}, h_{2 n-1}\right)\right] .
\end{aligned}
$$

If we suppose that $\theta\left[\sigma_{b}\left(h_{2 n-1}, h_{2 n}\right)\right] \leq \theta\left[\sigma_{b}\left(h_{2 n}, h_{2 n+1}\right)\right]$ for some $n \in \mathbb{N}$, then inequality (21) takes the form

$$
\begin{aligned}
\psi & \left(\sigma_{b}\left(h_{2 n+1}, h_{2 n}\right)\right) \\
& \leq \psi\left(s^{p} \sigma_{b}\left(h_{2 n+1}, h_{2 n}\right)\right) \\
& =\psi\left(s^{p} \sigma_{b}\left(f h_{2 n}, g h_{2 n-1}\right)\right) \\
& \leq\left(\beta\left[\sigma_{b}\left(h_{2 n}, h_{2 n-1}\right)\right]+\gamma\left[\sigma_{b}\left(h_{2 n}, h_{2 n-1}\right)\right]+\delta\left[\sigma_{b}\left(h_{2 n}, h_{2 n-1}\right)\right]\right) \theta\left[\sigma_{b}\left(h_{2 n}, h_{2 n+1}\right)\right] \\
& <\theta\left[\sigma_{b}\left(h_{2 n}, h_{2 n+1}\right)\right],
\end{aligned}
$$

that is, a contradiction. Hence

$$
\theta\left[\sigma_{b}\left(h_{2 n}, h_{2 n+1}\right)\right]<\theta\left[\sigma_{b}\left(h_{2 n-1}, h_{2 n}\right)\right] .
$$


By (22) and the properties of $\psi, \theta$, we get

$$
\psi\left[\sigma_{b}\left(h_{2 n+1}, h_{2 n}\right)\right] \leq \theta\left[\sigma_{b}\left(h_{2 n}, h_{2 n+1}\right)\right]<\theta\left[\sigma_{b}\left(h_{2 n-1}, h_{2 n}\right)\right]<\psi\left[\sigma_{b}\left(h_{2 n-1}, h_{2 n}\right)\right] .
$$

Inequality (23) implies $\sigma_{b}\left(h_{2 n}, h_{2 n+1}\right) \leq \sigma_{b}\left(h_{2 n-1}, h_{2 n}\right)$ for all $n \in \mathbb{N}$.

That is, the sequence $\left\{\sigma_{b}\left(h_{2 n+1}, h_{2 n}\right)\right\}$ is decreasing. Thus, it is convergent to $\inf \left\{\sigma_{b}\left(h_{2 n+1}\right.\right.$, $\left.\left.h_{2 n}\right)\right\}=r \geq 0$. That is, $\lim _{n \rightarrow \infty} \sigma_{b}\left(h_{n}, h_{n+1}\right)=r$, and also $\lim _{n \rightarrow \infty} \sigma_{b}\left(h_{2 n}, h_{2 n+1}\right)=$ $\lim _{n \rightarrow \infty} \sigma_{b}\left(h_{2 n-1}, h_{2 n}\right)=r$.

If we suppose $r>0$, then we consider

$$
\begin{aligned}
\psi\left(\sigma_{b}\left(h_{2 n+1}, h_{2 n}\right)\right) \leq & \psi\left(s^{p} \sigma_{b}\left(h_{2 n+1}, h_{2 n}\right)\right)=\psi\left(s^{p} \sigma_{b}\left(f h_{2 n}, g h_{2 n-1}\right)\right) \\
\leq & \psi\left(\alpha\left(h_{2 n}, h_{2 n-1}\right) \sigma_{b}\left(f h_{2 n}, g h_{2 n-1}\right)\right) \\
\leq & \beta\left[\sigma_{b}\left(h_{2 n}, h_{2 n-1}\right)\right] \theta\left[\sigma_{b}\left(h_{2 n}, h_{2 n-1}\right)\right] \\
& +\gamma\left[\sigma_{b}\left(h_{2 n}, h_{2 n-1}\right)\right] \theta\left[\sigma_{b}\left(h_{2 n}, h_{2 n+1}\right)\right] \\
& +\delta\left[\sigma_{b}\left(h_{2 n}, h_{2 n-1}\right)\right] \theta\left[\sigma_{b}\left(h_{2 n-1}, h_{2 n}\right)\right]
\end{aligned}
$$

and, letting $n \rightarrow \infty$ in (24), we obtain $\psi(r) \leq \theta(r)$, which implies that $r=0$, that is,

$$
\lim _{n \rightarrow \infty} \sigma_{b}\left(h_{n}, h_{n+1}\right)=\lim _{n \rightarrow \infty} \sigma_{b}\left(h_{n-1}, h_{n}\right)=0
$$

Now, we prove that $\lim _{n, m \rightarrow \infty} \sigma_{b}\left(h_{n}, h_{m}\right)=0$. It is sufficient to show that $\lim _{n, m \rightarrow \infty} \sigma_{b}\left(h_{2 n}\right.$, $\left.h_{2 m}\right)=0$. If we assume $\lim _{n, m \rightarrow \infty} \sigma_{b}\left(h_{2 n}, h_{2 m}\right) \neq 0$ then, using Lemma 1.11, there exists $\varepsilon>0$, and we can find subsequences $\left\{m_{i}\right\}$ and $\left\{n_{i}\right\}$ of positive integers, with $n_{i}>m_{i}>i$, such that

$$
\begin{aligned}
& \varepsilon \leq \lim _{i \rightarrow \infty} \sup _{i \rightarrow \infty} \sigma_{b}\left(h_{2 n_{i}}, h_{2 m_{i}}\right) \leq \varepsilon s, \quad \frac{\varepsilon}{s} \leq \lim \sup _{i \rightarrow \infty} \sigma_{b}\left(h_{2 m_{i}}, h_{2 n_{i}-1}\right) \leq \varepsilon s, \\
& \frac{\varepsilon}{s^{2}} \leq \lim \sup _{i \rightarrow \infty} \sigma_{b}\left(h_{2 n_{i}-1}, h_{2 m_{i}+1}\right) \leq \varepsilon s^{2}, \quad \frac{\varepsilon}{s} \leq \lim _{i \rightarrow \infty} \sup _{i \rightarrow \infty} \sigma_{b}\left(h_{2 m_{i}+1}, h_{2 n_{i}}\right) \leq \varepsilon s^{2} .
\end{aligned}
$$

Since $\alpha\left(h_{2 m_{i}}, h_{2 n_{i}-1}\right) \geq s^{p}$ from (19), we have

$$
\begin{aligned}
\psi\left(s^{p} \sigma_{b}\left(h_{2 m_{i}+1}, h_{2 n_{i}}\right)\right) \leq & \psi\left(s^{p} \sigma_{b}\left(f h_{2 m_{i}}, g h_{2 n_{i}-1}\right)\right) \\
\leq & \psi\left(\alpha\left(h_{2 m_{i}}, h_{2 n_{i}-1}\right) \sigma_{b}\left(f h_{2 m_{i}}, g h_{2 n_{i}-1}\right)\right) \\
\leq & \beta\left[\sigma_{b}\left(h_{2 m_{i}}, h_{2 n_{i}-1}\right)\right] \theta\left(\sigma_{b}\left(h_{2 m_{i}}, h_{2 n_{i}-1}\right)\right) \\
& +\gamma\left[\sigma_{b}\left(h_{2 m_{i}}, h_{2 n_{i}-1}\right)\right] \theta\left(\sigma_{b}\left(h_{2 m_{i}}, f h_{2 m_{i}}\right)\right) \\
& +\delta\left[\sigma_{b}\left(h_{2 m_{i}}, h_{2 n_{i}-1}\right)\right] \theta\left(\sigma_{b}\left(h_{2 n_{i}-1}, g h_{2 n_{i}-1}\right)\right) \\
= & \beta\left[\sigma_{b}\left(h_{2 m_{i}}, h_{2 n_{i}-1}\right)\right] \theta\left(\sigma_{b}\left(h_{2 m_{i}}, h_{2 n_{i}-1}\right)\right) \\
& +\gamma\left[\sigma_{b}\left(h_{2 m_{i}}, h_{2 n_{i}-1}\right)\right] \theta\left(\sigma_{b}\left(h_{2 m_{i}}, h_{2 m_{i}+1}\right)\right) \\
& +\delta\left[\sigma_{b}\left(h_{2 m_{i}}, h_{2 n_{i}-1}\right)\right] \theta\left(\sigma_{b}\left(h_{2 n_{i}-1}, h_{2 n_{i}}\right)\right) .
\end{aligned}
$$


Hence, by (26), (27), and (25), we obtain

$$
\begin{aligned}
\psi(\varepsilon s) \leq & \psi\left(\varepsilon s^{p-1}\right)=\psi\left(s^{p} \frac{\varepsilon}{s}\right) \leq \psi\left(\lim \sup _{i \rightarrow \infty} \sigma_{b}\left(h_{m_{i}}, h_{n_{i}}\right)\right) \\
\leq & \lim \sup _{i \rightarrow \infty} \beta\left[\sigma_{b}\left(h_{2 m_{i}}, h_{2 n_{i}-1}\right)\right] \theta\left(\lim \sup _{i \rightarrow \infty} \sigma_{b}\left(h_{2 m_{i}}, h_{2 n_{i}-1}\right)\right) \\
& +\lim \sup _{i \rightarrow \infty} \gamma\left[\sigma_{b}\left(h_{2 m_{i}}, h_{2 n_{i}-1}\right)\right] \theta\left(\lim \sup _{i \rightarrow \infty} \sigma_{b}\left(h_{2 m_{i}}, h_{2 m_{i}+1}\right)\right) \\
& +\lim \sup _{i \rightarrow \infty} \delta\left[\sigma_{b}\left(h_{2 m_{i}}, h_{2 n_{i}-1}\right)\right] \theta\left(\lim \sup _{i \rightarrow \infty} \sigma_{b}\left(h_{2 n_{i}-1}, h_{2 n_{i}}\right)\right) \\
= & \lim \sup _{i \rightarrow \infty} \beta\left[\sigma_{b}\left(h_{2 m_{i}}, h_{2 n_{i}-1}\right)\right] \theta(\varepsilon s)+\limsup _{i \rightarrow \infty} \gamma\left[\sigma_{b}\left(h_{2 m_{i}}, h_{2 n_{i}-1}\right)\right] \theta(0) \\
& +\lim \sup _{i \rightarrow \infty} \delta\left[\sigma_{b}\left(h_{2 m_{i}}, h_{2 n_{i}-1}\right)\right] \theta(0) \\
\leq & \theta(\varepsilon s),
\end{aligned}
$$

which implies $\varepsilon=0$, a contradiction. Thus, $\lim _{n, m \rightarrow \infty} \sigma_{b}\left(h_{n}, h_{m}\right)=0$, and the sequence $\left\{h_{n}\right\}$ is Cauchy. $\left(T, \sigma_{b}\right)$ is complete, so there exists $h \in T$ such that $\left\{h_{n}\right\}$ is convergent to $h$, that is,

$$
\lim _{n \rightarrow \infty} \sigma_{b}\left(h_{n}, h\right)=\lim _{n \rightarrow \infty} \sigma_{b}\left(h_{n}, h_{m}\right)=\sigma_{b}(h, h)=0 .
$$

By property $H_{s}$, there exists a subsequence $\left\{h_{n_{i}}\right\}$ of $\left\{h_{n}\right\}$ with $\alpha\left(h_{n_{i}}, h\right) \geq s^{p}$ and $\alpha\left(h, h_{n_{i}}\right) \geq$ $s^{p}$ for all $i \in \mathbb{N}$. Then, from condition (19), we have

$$
\begin{aligned}
\psi\left(s^{p} \sigma_{b}\left(h_{2 n_{i}+1}, g h\right)\right)= & \psi\left(s^{p} \sigma_{b}\left(f h_{2 n(i)}, g h\right)\right) \leq \psi\left(\alpha\left(h_{2 n(i)}, h\right) \sigma_{b}\left(f h_{2 n(i)}, g h\right)\right) \\
\leq & \beta\left[\sigma_{b}\left(h_{2 n(i)}, h\right)\right] \theta\left(\sigma_{b}\left(h_{2 n(i)}, h\right)\right) \\
& +\gamma\left[\sigma_{b}\left(h_{2 n(i)}, h\right)\right] \theta\left(\sigma_{b}\left(h_{2 n(i)}, f h_{2 n(i)}\right)\right) \\
& +\delta\left[\sigma_{b}\left(h_{2 n(i)}, h\right)\right] \theta\left(\sigma_{b}(h, g h)\right) \\
= & \beta\left[\sigma_{b}\left(h_{2 n_{i}}, h\right)\right] \theta\left(\sigma_{b}\left(h_{2 n_{i}}, h\right)\right) \\
& +\gamma\left[\sigma_{b}\left(h_{2 n_{i}}, h\right)\right] \theta\left(\sigma_{b}\left(h_{2 n_{i}}, h_{2 n_{i}+1}\right)\right) \\
& +\delta\left[\sigma_{b}\left(h_{2 n_{i}}, h\right)\right] \theta\left(\sigma_{b}(h, g h)\right) .
\end{aligned}
$$

Considering limit superior as $i \rightarrow \infty$ in (29), and due to (25), (28), and Lemma 1.9, we obtain

$$
\begin{aligned}
\psi\left(s^{p-1} \sigma_{b}(h, g h)\right)= & \psi\left(s^{p} s^{-1} \sigma_{b}(h, g h)\right) \leq \psi\left(s^{p} \lim \sup _{i \rightarrow \infty} \sigma_{b}\left(h_{2 n_{i}+1}, g h\right)\right) \\
\leq & \lim \sup _{i \rightarrow \infty} \beta\left[\sigma_{b}\left(h_{2 n_{i}}, h\right)\right] \theta\left(\lim \sup _{i \rightarrow \infty} \sigma_{b}\left(h_{2 n_{i}}, h\right)\right) \\
& +\lim \sup _{i \rightarrow \infty} \gamma\left[\sigma_{b}\left(h_{2 n_{i}}, h\right)\right] \theta\left(\lim \sup _{i \rightarrow \infty} \sigma_{b}\left(h_{2 n_{i}}, h_{2 n_{i}+1}\right)\right) \\
& +\lim \sup _{i \rightarrow \infty} \delta\left[\sigma_{b}\left(h_{2 n_{i}}, h\right)\right] \theta\left(\lim \sup _{i \rightarrow \infty} \sigma_{b}(h, g h)\right) \\
\leq & \theta\left(\sigma_{b}(h, g h)\right) .
\end{aligned}
$$


Inequality (30) yields that $\sigma_{b}(h, g h)=0$, so $g h=h$. Similarly, $f h=h$.

If $h, j \in C(f, g)$ with $h \neq j$, then, by hypothesis $U_{s^{p}}$ and applying (19), we obtain

$$
\begin{aligned}
\psi & \left(s^{p} \sigma_{b}(h, h)\right) \\
& \leq \psi\left(\alpha(h, h) \sigma_{b}(f h, g h)\right) \\
& \leq \beta\left[\sigma_{b}(h, h)\right] \theta\left(\sigma_{b}(h, h)\right)+\gamma\left[\sigma_{b}(h, h)\right] \theta\left(\sigma_{b}(h, f h)\right)+\delta\left[\sigma_{b}(h, h)\right] \theta\left(\sigma_{b}(h, g h)\right) \\
& =\beta\left[\sigma_{b}(h, h)\right] \theta\left(\sigma_{b}(h, h)\right)+\gamma\left[\sigma_{b}(h, h)\right] \theta\left(\sigma_{b}(h, h)\right)+\delta\left[\sigma_{b}(h, h)\right] \theta\left(\sigma_{b}(h, h)\right) \\
& =\left(\beta\left[\sigma_{b}(h, h)\right]+\gamma\left[\sigma_{b}(h, h)\right]+\delta\left[\sigma_{b}(h, h)\right]\right) \theta\left(\sigma_{b}(h, h)\right) \\
& <\theta\left(\sigma_{b}(h, h)\right),
\end{aligned}
$$

that implies $\sigma_{b}(h, h)=0\left(\right.$ also $\left.\sigma_{b}(j, j)=0\right)$.

Again from (19), we have

$$
\begin{aligned}
\psi\left(s^{p} \sigma_{b}(h, j)\right) \leq & \psi\left(\alpha(h, j) \sigma_{b}(f h, g j)\right) \\
\leq & \beta\left[\sigma_{b}(h, j)\right] \theta\left(\sigma_{b}(h, j)\right)+\gamma\left[\sigma_{b}(h, j)\right] \theta\left(\sigma_{b}(h, f h)\right) \\
& +\delta\left[\sigma_{b}(h, j)\right] \theta\left(\sigma_{b}(j, g j)\right) \\
\leq & \theta\left(\sigma_{b}(h, j)\right),
\end{aligned}
$$

a contradiction. Hence, $h=j$.

Corollary 2.11 Let $(f, g)$ be an $\alpha_{s^{p}}$-admissible pair of self-mappings on a complete $b$ metric-like space $\left(T, \sigma_{b}\right)$ with coefficient $s \geq 1$. If there exist $\psi \in I, \theta \in \Theta$ and $c_{1}, c_{2}, c_{3} \in \mathbb{R}^{+}$ with $c_{1}+c_{2}+c_{3}<1$ such that

$$
\psi\left(\alpha(h, k) \sigma_{b}(f h, g k)\right) \leq c_{1} \theta\left[\sigma_{b}(h, k)\right]+c_{2} \theta\left[\sigma_{b}(h, f h)\right]+c_{3} \theta\left[\sigma_{b}(k, g k)\right]
$$

for all $h, k \in T$; furthermore, the following conditions hold:

(i) there exists $h_{0} \in T$ such that $\min \left\{\alpha\left(h_{0}, f h_{0}\right), \alpha\left(f h_{0}, h_{0}\right)\right\} \geq s^{p}$;

(ii) properties $H_{s^{p}} ; U_{s^{p}}$ are satisfied,

then $f$ and $g$ have a unique common fixed point $h \in T$.

Proof Take in Theorem 2.10, $\beta(m)=c_{1}, \gamma(m)=c_{2}, \delta(m)=c_{3}, m \geq 0$.

Corollary 2.12 Let $f$ be an $\alpha_{s^{p}}$-admissible self-mapping on a b-metric-like space $\left(T, \sigma_{b}\right)$ with coefficient $s \geq 1$. Iff is a generalized $\alpha_{s} p-(I \times \Theta \times \Gamma)$-Kannan contraction, and the following assertions hold:

(i) there exists $h_{0} \in T$ such that $\min \left\{\alpha\left(h_{0}, f h_{0}\right), \alpha\left(f h_{0}, h_{0}\right)\right\} \geq s^{p}$;

(ii) conditions $H_{s^{p}}$; $U_{s^{p}}$ are satisfied, then $f$ has a unique fixed point $h \in T$.

Proof The proof follows from Theorem 2.10 if we take $g=f$.

Corollary 2.13 Let $(f, g)$ be an $\alpha_{s^{p}}$-admissible pair of self-mappings on a complete $b$ metric-like space $\left(T, \sigma_{b}\right)$ with coefficient $s \geq 1$. If there exist $\psi \in I, \theta \in \Theta$, and $\beta \in \Gamma$ such 
that

$$
\psi\left(\alpha(h, k) \sigma_{b}(f h, g k)\right) \leq \beta\left[\sigma_{b}(h, k)\right]\left(\theta\left[\sigma_{b}(h, k)\right]+\theta\left[\sigma_{b}(h, f h)\right]+\theta\left[\sigma_{b}(k, g k)\right]\right)
$$

for all $h, k \in T$ and $\theta(m)<\psi(m)$ for all $m>0$; and the following assertions hold:

(i) there exists $h_{0} \in T$ such that $\min \left\{\alpha\left(h_{0}, f h_{0}\right), \alpha\left(f h_{0}, h_{0}\right)\right\} \geq s^{p}$;

(ii) conditions $H_{s^{p}} ; U_{s^{p}}$ are satisfied,

then $f$ and $g$ have a unique common fixed point $h \in T$.

Proof By taking $\gamma(m)=\delta(m)=\beta(m)$.

Remark 2.14 It is evident that we can generate a variety of other corollaries as special cases by putting $\alpha(h, k)=s^{p}(p>1)$, or $g=f$ or $\psi(m)=m$, or defining $\beta, \gamma, \delta \in \Gamma$ as constant functions.

\section{Applications}

In this section, we discuss an application that attributes the solvability of boundary value problems of second order ordinary differential equations:

$$
\begin{cases}h^{\prime \prime}(u)=M_{1}(u, h(u)), & u \in[0,1], \\ h^{\prime \prime}(u)=M_{2}(u, h(u)), & u \in[0,1], \\ h(0)=h(1)=0 & \end{cases}
$$

for given continuous functions $M_{1} ; M_{2}:[0,1] \times \mathbb{R} \rightarrow \mathbb{R}$.

Let $T=\complement([0,1], \mathbb{R})$ be the set of real continuous functions defined on $[0,1]$, endowed with the $b$-metric-like

$$
\sigma_{b}(h, k)=\max _{u \in[0,1]}(|h(u)|+|k(u)|)^{n} \quad \text { for all } h, k \in T .
$$

It is evident that $\left(T, \sigma_{b}\right)$ is a complete $b$-metric-like space with parameter $s=2^{n-1}$ where $n>1$.

The equivalent system of integral equations corresponding to boundary value problems (31) is the following:

$$
\left\{\begin{array}{c}
h(u)=\int_{0}^{1} G(u, \rho) M_{1}(\rho, h(\rho)) d \rho, \\
h(u)=\int_{0}^{1} G(u, \rho) M_{2}(\rho, h(\rho)) d \rho, \\
\text { for } u \in[0,1]
\end{array}\right.
$$

and $G(u, \rho)$ is the Green function given as

$$
G(u, \rho)= \begin{cases}\rho(u-\rho) & 0 \leq \rho \leq u \leq 1 \\ u(\rho-u) & 0 \leq u \leq \rho \leq 1\end{cases}
$$

Consider the mappings $f, g: T \rightarrow T$ by

$$
f h(u)=\int_{0}^{1} G(u, \rho) M_{1}(\rho, h(\rho)) d \rho \quad \text { for } u \in[0,1]
$$




$$
g h(u)=\int_{0}^{1} G(u, \rho) M_{2}(\rho, h(\rho)) d \rho \quad \text { for } u \in[0,1],
$$

and let $\zeta: \mathbb{R} \times \mathbb{R} \rightarrow \mathbb{R}$ be a given function.

Theorem 3.1 Consider the system of integral Eqs. (32) and suppose that the following assertions hold:

(i) There exists $h_{0} \in T$ such that $\zeta\left(h_{0}(u), f h_{0}(u)\right) \geq 0$ for all $u \in[0,1]$;

(ii) For all $u \in[0,1]$ and $h, k \in T$,

$$
\zeta(h(u), k(u)) \geq 0 \quad \text { implies that } \zeta(f h(u), g k(u)) \geq 0 \text {; }
$$

(iii) Properties $H_{s^{p}}$ and $U_{s^{p}}$ are satisfied;

(iv) There exist $n>1, p>1, \lambda \in(0,1)$ and a continuous function $\theta: \mathbb{R}^{+} \rightarrow \mathbb{R}^{+}$such that

$$
\left(\left|M_{1}(\rho, h(\rho))\right|+\left|M_{2}(\rho, h(\rho))\right|\right) \leq \sqrt[n]{L 2^{p} \theta\left[(|h(\rho)|+|k(\rho)|)^{n}\right]}
$$

for all $\rho \in[0,1], h, k \in T$;

(v) For all $\rho \in[0,1], \sup _{u \in[0,1]} \int_{0}^{1} G(u, \rho) d \rho \leq \frac{1}{2}$.

Then the system of integral Eqs. (32) (or equivalently, (31)) has a unique solution in T.

Proof We define a function $\alpha: T \times T \rightarrow[0, \infty)$ by

$$
\alpha(h, k)= \begin{cases}s^{p} & \text { if } \zeta(h(u), k(u)) \geq 0, \text { for all } u \in[0,1] \\ 0 & \text { otherwise. }\end{cases}
$$

It is clear that $(f, g)$ is an $\alpha_{s^{p}}$-admissible pair.

Let $h, k \in T=\complement([0,1], \mathbb{R})$ be such that $\alpha(h(u), k(u)) \geq s^{p}$, i.e., $\zeta(f h(u), g k(u)) \geq 0$, then from the assertions above, for all $u \in[0,1]$, we might observe that

$$
\begin{aligned}
\sigma_{b}(f h(u), g k(u)) & =\max _{u \in[0,1]}(|f h(u)|+|g k(u)|)^{n} \\
& =\left(\left|\int_{0}^{1} G(u, \rho) M_{1}(\rho, h(\rho)) d \rho\right|+\left|\int_{0}^{1} G(u, \rho) M_{2}(\rho, h(\rho)) d \rho\right|\right)^{n} \\
& \leq\left(\int_{0}^{1} G(u, \rho)\left|M_{1}(\rho, h(\rho))\right| d \rho+\int_{0}^{1} G(u, \rho)\left|M_{2}(\rho, h(\rho))\right| d \rho\right)^{n} \\
& =\left(\int_{0}^{1} G(u, \rho)\left(\left|M_{1}(\rho, h(\rho))\right|+\left|M_{2}(\rho, h(\rho))\right|\right) d \rho\right)^{n} \\
& \leq\left(\int_{0}^{1} G(u, \rho) \sqrt[n]{\lambda 2^{p} \theta\left[(|h(\rho)|+|k(\rho)|)^{n}\right]} d \rho\right)^{n} \\
& =\left(\int_{0}^{1} G(u, \rho) \sqrt[n]{\lambda 2^{p} \theta\left[\sigma_{b}(h(\rho), k(\rho))\right]} d \rho\right)^{n} \\
& =\left(\int_{0}^{1} G(u, \rho) d \rho\right)^{n} \lambda 2^{p} \theta\left[\sigma_{b}(h(\rho), k(\rho))\right] \\
& \leq\left(\sup _{u \in[0,1]} \int_{0}^{1} G(u, \rho) d \rho\right)^{n} \lambda 2^{p} \theta\left[\sigma_{b}(h, k)\right] .
\end{aligned}
$$


Since $G(u, \rho)=\frac{u}{2}-\frac{u^{2}}{2}$ and $\sup _{u \in[0,1]} \int_{0}^{1} G(u, \rho) d \rho \leq \frac{1}{8}$ (in that case, the coefficient $p=3$ > $1)$, then inequality (33) can be written

$$
\sigma_{b}(f h(u), g k(u)) \leq \frac{1}{2^{n p}} \cdot \frac{\lambda}{2^{-p}} \theta\left[\sigma_{b}(h, k)\right] \leq \frac{\lambda}{2^{(n-1) p}} \theta\left[\sigma_{b}(h, k)\right]=\frac{\lambda}{s^{p}} \theta\left[\sigma_{b}(h, k)\right] .
$$

Hence,

$$
\max _{u \in[0,1]}(|f h(u)|+|g k(u)|)^{n} \leq \frac{\lambda}{s^{p}} \theta\left[\sigma_{b}(h, k)\right]
$$

and we convert the result to

$$
\alpha(h, k) \sigma_{b}(f h(u), g k(u)) \leq \lambda \theta\left[\sigma_{b}(h, k)\right] .
$$

Thus, taking $\psi(x)=x$, and $\beta, \gamma, \delta \in \Gamma$ as $\beta(x)=\lambda, \gamma(x)=0, \delta(x)=0$, where $\lambda \in(0,1)$, from inequality (34) we deduce

$$
\begin{aligned}
\psi\left(\alpha(h, k) \sigma_{b}(f h, g k)\right) \leq & \beta\left[\sigma_{b}(h, k)\right] \theta\left[\sigma_{b}(h, k)\right] \\
& +\gamma\left[\sigma_{b}(h, k)\right] \theta\left[\sigma_{b}(h, f h)\right] \\
& +\delta\left[\sigma_{b}(h, k)\right] \theta\left[\sigma_{b}(k, g k)\right] .
\end{aligned}
$$

Therefore, Theorem 2.10 can be applied to obtain a solution of the system of boundary value problems (31).

\section{Acknowledgements}

The authors extend their appreciation to the Deanship of Post Graduate and Scientific Research at Dar Al Uloom University for funding this work.

\section{Funding}

No funding sources to be declared.

Availability of data and materials

Please contact the authors for data request.

\section{Competing interests}

The authors declare that they have no competing interests.

\section{Authors' contributions}

The authors contributed equally to this work. All authors read and approved the final manuscript.

\section{Author details}

'Department of Mathematics and Computer Sciences, Faculty of Natural Sciences, University of Gjirokastra, Gjirokastra 6001, Albania. ${ }^{2}$ Institut Supérieur d'Informatique et des Techniques de Communication, Université de Sousse, H. Sousse, 4000, Tunisia. ${ }^{3}$ Department of Mathematics and Applied Mathematics, Sefako Makgatho Health Sciences University, Ga-Rankuwa, South Africa. ${ }^{4}$ China Medical University Hospital, China Medical University, Taichung, 40402, Taiwan. ${ }^{5}$ College of Business Administration-Finance Department, Dar Al Uloom University Saudi Arabia, Riyadh, Saudi Arabia.

\section{Publisher's Note}

Springer Nature remains neutral with regard to jurisdictional claims in published maps and institutional affiliations.

Received: 11 January 2021 Accepted: 5 May 2021 Published online: 19 May 2021

\section{References}

1. Younis, M., Singh, D., Asadi, M., Joshi, V.: Results on contractions of Reich type in graphical $b$-metric spaces with applications. Filomat 33(17), 5723-5735 (2019)

2. Mlaiki, N., Aydi, H., Souayah, N., Abdeljawad, T.: Controlled metric type spaces and the related contraction principle. Mathematics 6(10), 194 (2018) 
3. Aydi, H., Chen, C.M., Karapinar, E.. Interpolative Ciric-Reich-Rus type contractions via the Branciari distance. Mathematics 7(1), 84 (2019)

4. Alghamdi, M., Ozyurt, S.G., Karapinar, E.: A note on extended Z-contraction. Mathematics 8(2), 195 (2020)

5. Marasi, H.R., Aydi, H.: Existence and uniqueness results for two-term nonlinear fractional differential equations via a fixed point technique. J. Math. 2021, Article ID 6670176 (2021)

6. Karapinar, E., Chifu, C.: Results in wt-distance over b-metric spaces. Mathematics 8, 195 (2020)

7. Ameer, E., Aydi, H., Arshad, M., De la Sen, M.: Hybrid Ćirić type graphic $(\Upsilon, \Lambda)$-contraction mappings with applications to electric circuit and fractional differential equations. Symmetry 12(3), 467 (2020)

8. Karapinar, E., Fulga, A., Petrusel, P.: On Isatratscu type contractions in b-metric spaces. Mathematics 8, 388 (2020)

9. Patle, P., Patel, D., Aydi, H., Radenovic, S.: On $\mathrm{H}^{+}$-type multivalued contractions and applications in symmetric and probabilistic spaces. Mathematics 7(2), 144 (2019)

10. Karapinar, E., Chifu, C.: Admissible hybrid Z-contractions in b-metric spaces. Axioms 9, 2 (2020)

11. Parvaneh, V., Haddadi, M.R., Aydi, H.: On best proximity point results for some type of mappings. J. Funct. Spaces 2020, Article ID 6298138 (2020)

12. Eshraghisamani, M., Vaezpour, M.S., Asadi, M.: New fixed point results with $\alpha_{q s} p$-admissible contractions on b-Branciari metric spaces. J. Inequal. Spec. Funct. 9(4), 38-46 (2018)

13. Zoto, K., Radenovic, S., Dine, J., Vardhami, I.: Fixed points of generalized $(\psi, \mathrm{s}, \alpha)$-contractive mappings in dislocated and b-dislocated metric spaces. Commun. Optim. Theory 2017, 1 (2017)

14. Zoto, K., Vardhami, I.: Common fixed point results for generalized $\alpha_{s p}$-contractive mappings and applications. J. Funct. Spaces 2018, Article ID 1282414 (2018)

15. Zoto, K., Rhoades, B.E., Radenovic, S.: Common fixed point theorems for a class of $(s, q)$-contractive mappings in b-metric-like spaces and applications to integral equations. Math. Slovaca 69(1), 1-15 (2019)

16. Zoto, K., Radenovic, S., Ansari, A.H.: On some fixed point results for $(s, p, \alpha)$-contractive mappings in b-metric-like spaces and applications to integral equations. Open Math. 16, 235-249 (2018)

17. Afshari, H., Aydi, H., Karapınar, E.: On generalized $\alpha-\psi$-Geraghty contractions on b-metric spaces. Georgian Math. J. 27, 9-21 (2020)

18. Karapinar, E., Czerwik, S., Aydi, H.: $(\alpha, \psi)$-Meir-Keeler contraction mappings in generalized b-metric spaces. J. Funct. Spaces 2018, Article ID 3264620 (2018)

19. Eshraghisamani, M., Vaezpour, M.S., Asadi, M.: New fixed point results on Branciari metric spaces. J. Math. Anal. 8(6), 132-141 (2017)

20. Aydi, H., Lakzian, H., Mitrovic, Z.D., Radenovic, S.: Best proximity points of MF-cyclic contractions with property UC. Numer. Funct. Anal. Optim. 41(7), 871-882 (2020)

21. Zoto, K., Rhoades, B.E., Radenovic, S.: Some generalizations for $(\alpha-\psi, \varphi)$ contractions in b-metric-like spaces and an application. Fixed Point Theory Appl. 2017, 26 (2017)

22. Hussain, N., Radenovic, S., Zoto, K.: Common fixed point results of $(\alpha-\psi, \varphi)$-contractions for a pair of mappings and applications. Mathematics 6, 182 (2018)

23. Zoto, K., Mlaiki, N., Aydi, H.: Related fixed point theorems via general approach of simulations functions. J. Math. 2020 Article ID 4820191 (2020)

24. Matthews, S.G.: Partial metric topology. In: Proc. 8th Summer Conference on General Topology and Applications, Ann. New York Acad. Sci., vol. 728, pp. 183-197 (1994)

25. Hitzler, P., Seda, A.K.: Dislocated topologies. J. Electr. Eng. 51(12), 3-7 (2000)

26. Amini-Harandi, A.: Metric-like spaces, partial metric spaces and fixed points. Fixed Point Theory Appl. 2012, 204 (2012)

27. Alghamdi, M.A., Hussain, N., Salimi, P.: Fixed point and coupled fixed point theorems on b-metric-like spaces. J. Inequal. Appl. 2013, 402 (2013)

28. Czerwik, S.: Contraction mappings in b-metric spaces. Acta Math. Inform. Univ. Ostrav. 1, 5-11 (1993)

29. Samet, B., Vetro, C., Vetro, P.: Fixed point theorems for $\alpha-\psi$-contractive type mappings. Nonlinear Anal. 75 2154-2165 (2012)

30. Aydi, $\mathrm{H} .: \alpha$-Implicit contractive pair of mappings on quasi b-metric spaces and an application to integral equations. J. Nonlinear Convex Anal. 17(12), 2417-2433 (2016)

31. Hoxha, E., Ansari, A.H., Zoto, K.: Some common fixed point results through generalized altering distances on dislocated metric spaces. In: Proceedings of EllC, September 1-5, pp. 403-409 (2014)

\section{Submit your manuscript to a SpringerOpen ${ }^{\circ}$ journal and benefit from:}

- Convenient online submission

- Rigorous peer review

- Open access: articles freely available online

- High visibility within the field

- Retaining the copyright to your article

Submit your next manuscript at $\gg$ springeropen.com 